\title{
INFLUÊNCIA DA COMBINAÇÃO DE DADOS GPS E GLONASS NO GEORREFERENCIAMENTO DE IMÓVEIS RURAIS
}

\author{
Influence of combination of GPS and GLONASS data in georeferencing of rural \\ properties \\ MARCELO SOLFA PINTO ${ }^{1}$ \\ PAULO DE OLIVEIRA CAMARGO ${ }^{2}$ \\ JOÃO FRANCISCO GALERA MONICO ${ }^{2}$ \\ 1,2 Programa de Pós-Graduação em Ciências Cartográficas \\ ${ }^{2}$ Departamento de Cartografia - Faculdade de Ciências e Tecnologia/Universidade \\ Estadual Paulista - FCT/Unesp \\ marcelosolfa@gmail.com; \{paulo; galera\}@fct.unesp.br
}

\begin{abstract}
RESUMO
A questão agrária ainda é um tema bastante discutido no Brasil, principalmente no que diz respeito à posse da terra. A Lei $\mathrm{n}^{\circ} 10.267 / 01$ foi um grande avanço para a questão agrária. A partir de então, todos os imóveis rurais devem ser georreferenciados ao Sistema Geodésico Brasileiro (SGB). Para isso, são utilizados métodos de posicionamento por satélites e convencionais. Mudanças vêm ocorrendo nos sistemas de posicionamento por satélites em razão do acréscimo de novos sinais no GPS (Global Positionig System) e a reestruturação do GLONASS (Global Orbiting Navigation Satellite System), além dos novos sistemas, Galileo e Compass. Para avaliar os efeitos da combinação de dados GPS e GLONASS, foram realizados processamentos de dados com diferentes configurações. Os processamentos foram realizados para a determinação das coordenadas dos pontos de apoio básico e limítrofes de imóveis rurais. Ao final, verificou-se que o uso de efemérides precisas no transporte de coordenadas de pontos de apoio não apresenta influência relevante, pois o transporte com efemérides transmitidas também atende a exigência de precisão da Norma Técnica de Georreferenciamento de Imóveis Rurais (NTGIR). Quando foram utilizados dados GPS e GLONASS, as melhores soluções foram obtidas. No caso dos pontos limítrofes, o uso de dados GPS e GLONASS também é recomendado, pois atende a exigência de precisão da NTGIR e apresenta resultados melhores que dos dados processados separadamente.
\end{abstract}


Palavras-chave: Georreferenciamento; Imóveis Rurais; GPS/GLONASS; Integração de Sistemas.

\begin{abstract}
The land question has been a widely discussed topic in Brazil, regarding land tenure. Law No. 10.267/01 was a major breakthrough for the agrarian issue. Since then on all rural properties must be georeferenced to the Brazilian Geodetic System (BGS). Therefore, satellite positioning and conventional methods are extensively used. Changes have been occurring in satellite positioning systems due to the addition of new signals in GPS (Global System Positioning), restructuring of GLONASS (Global Orbiting Navigation Satellite System), and the new systems like Galileo and Compass as well. To evaluate the effects of combining GPS and GLONASS data, several batches of processings were performed on different configurations. The data processing was performed to determine the coordinates of points of basic support and those materializing the neighborhood of the rural properties. As a result, it was found that the use of accurate ephemeris in transporting coordinates to support points has no significant influence, since transportation with broadcast ephemeris also meets the accuracy requirements for the Standard Technique for Georreferencing Rural Properties. On the other hand, when GPS and GLONASS data were used, such combination provides the best results. In the case of neighboring points, the use of GPS and GLONASS data is also recommended because such data meet the precision requirement and showed better results than those from where data were processed separately.
\end{abstract}

Keywords: Georreferencing; Rural Properties; GPS/GLONASS; System Integration.

\title{
1. INTRODUÇÃO
}

O GNSS (Global Navigation Sattelite System - Sistema Global de Navegação por Satélite) é constituído por diferentes sistemas de posicionamento por satélites. Dentre os sistemas que compõem o GNSS pode-se destacar o GPS (Global Positioning System), o GLONASS (Global Orbiting Navigation Sattelite System), o Galileo e o Beidou/Compass, bem como os sistemas de aumento como WAAS (Wide Area Augmentation System - USA), EGNOS (European Geostationary Navigation Overlay Service), o GAGAN (GPS Aided Geo Augmented Navigation India), MSAS (Multi-functional Satellite Augmentation System - Japão). O GNSS tem potencialidade de aplicações em várias áreas do conhecimento, quer seja em nível científico ou prático.

No Brasil, dentre as várias aplicações em que o GNSS é utilizado, destaca-se o georreferenciamento de áreas rurais, que visa o cadastro dos imóveis rurais, regulamentado pela Lei 10.267/01 (BRASIL, 2001). Além do georreferenciamento, outras aplicações podem ser destacadas, tais como a demarcação de Reserva Legal (RL) e Área de Preservação Permanente (APP). Outra aplicação que vem ganhando 
espaço no Brasil é a agricultura de precisão, na qual receptores GNSS são utilizados como um componente para o controle de máquinas em campo, mapeamento de produtividade, pulverização de fertilizantes e outras atividades.

Tendo em vista as aplicações dos sistemas GNSS para mapeamento de áreas rurais e de conservação, pretende-se avaliar neste trabalho o desempenho da combinação das observáveis dos sistemas GPS e GLONASS no georreferenciamento de imóveis rurais.

Para tanto, foram realizados levantamentos de campo utilizando receptores GNSS com capacidade de rastrear dados GPS e GLONASS. Os experimentos foram realizados em uma área teste localizada no Assentamento São Jorge, administrado pelo ITESP (Instituto de Terras do Estado de São Paulo) no município de Presidente Bernardes - SP. Esta área teste foi estabelecida em parceria entre a FCT/UNESP e o ITESP (LEITE et al., 2005), e possui uma área de 1.115 hectares, a qual foi subdividida em três áreas, simulando três propriedades rurais a serem levantadas.

\section{ESTADO DA ARTE EM GERENCIAMENTO DE TERRAS NO BRASIL}

No Brasil, o registro de imóveis rurais era e ainda é separado do cadastro. Porém, com a Lei 10.267/01, de 28 de agosto de 2001 (BRASIL, 2001), pretende-se a união entre o registro e o cadastro; formando uma única base de dados do imóvel, fato este ainda não ocorrido.

A lei tem como objetivo o combate à grilagem de terras e a formação ilegal de latifúndios, mas também cria o CNIR (Cadastro Nacional de Imóveis Rurais), que é um grande avanço na cartografia cadastral brasileira, pois gera um banco de dados territoriais georreferenciados, constituindo uma ferramenta de grande importância para a gestão territorial, planejamento e desenvolvimento do país.

\subsection{Legislação e Decretos}

A Lei 10.267/01 (BRASIL, 2001) determina que a identificação, a localização, os limites e os confrontantes das propriedades sejam obtidos a partir de memorial descritivo, o qual deve conter as coordenadas dos vértices definidores da propriedade georreferenciada ao SGB. A precisão posicional estabelecida para as coordenadas deve ser igual, ou melhor, do que $0,50 \mathrm{~m}$ (1 sigma), conforme o decreto normativo $\mathrm{n}^{\circ} 4.449 / 02$ (BRASIL, 2002). O decreto atribui ao INCRA a competência de certificar se o polígono objeto do memorial descritivo não se sobrepõe a nenhuma outra propriedade que conste de seu cadastro georreferenciado, e se atende à precisão estabelecida.

Esta lei determinou a criação do CNIR, que terá uma base comum de informações, gerenciada pelo INCRA e pela Secretaria da Receita Federal, produzida e compartilhada por diversas instituições públicas federais e estaduais, produtoras e usuárias de informação sobre o meio rural brasileiro.

O decreto $n^{\circ} 4.449$, de 30 de outubro de 2002 (BRASIL, 2002), regulamenta a Lei 10.267/01, e atribui ao INCRA e a Secretaria da Receita Federal o estabelecimento dos critérios técnicos para implementação, gerenciamento e 
alimentação do CNIR, bem como estabelece os prazos para a identificação da área do imóvel rural. O artigo $9^{\circ}$ estabelece que a identificação do imóvel rural seja obtida a partir de memorial descritivo elaborado, executado e assinado por profissional habilitado e com a devida ART (Anotação de Responsabilidade Técnica), contendo as coordenadas dos vértices definidores dos limites dos imóveis rurais, georreferenciadas ao SGB, determinadas de acordo com a Norma Técnica elaborada pelo INCRA.

Em 17 de novembro de 2003 publicou-se a $1^{\text {a }}$ edição da Norma Técnica para Georreferenciamento de Imóveis Rurais (INCRA, 2003). Recentemente a $2^{\mathrm{a}}$ edição revisada e publicada em 22 de fevereiro de 2010 (INCRA, 2011).

A identificação da área do imóvel rural, ou seja, o georreferenciamento imediato é exigido nos casos de desmembramento, parcelamento, remembramento e em qualquer situação de transferência de imóvel rural. O Decreto $n^{\circ}$ 5.570/05 (BRASIL, 2005), dá nova redação aos dispositivos do Decreto $n^{\circ} 4.449 / 02$, estabelecendo novos prazos para o georreferenciamento dos imóveis, no entanto, um novo decreto, Decreto $n^{\circ} 7.620 / 11$ estabelece novos prazos para áreas menores que quinhentos hectares a partir de 21 de novembro de 2011:

I - dez anos, para os imóveis com área de duzentos e cinquenta a menos de quinhentos hectares;

II - treze anos, para os imóveis com área de cem a menos de duzentos e cinquenta hectares;

III - dezesseis anos, para os imóveis com área de vinte e cinco a menos de cem hectares; e

IV - vinte anos, para os imóveis com área inferior a vinte e cinco hectares.

\subsection{Situação atual e estrutura de apoio aos levantamentos}

O Brasil possui, aproximadamente, 5,5 milhões de imóveis rurais e, até janeiro de 2013, somente $0,8 \%$ desses imóveis foram georreferenciados e certificados pelo INCRA. Trata-se de um número muito pequeno considerando a quantidade de propriedades; no entanto, as áreas dessas propriedades representam grande parte do território nacional, conforme apresentado pela Figura 1. Segundo INCRA (2013), existem aproximadamente 45.000 propriedades certificadas e 6.040 profissionais habilitados a realizarem o georreferenciamento em todo o Brasil.

Em relação à estrutura geodésica para apoio aos trabalhos de georreferenciamento, pode-se citar como exemplo, a RBMC (Rede Brasileira de Monitoramento Contínuo), que também disponibiliza dados da RIBaC (Rede INCRA de Bases Comunitárias) e da Rede GNSS SP da FCT/UNESP, as redes estaduais constituída de pontos passivos, num total 13, abrangem 18 estados. A rede Nordeste envolve 5 estados (IBGE, 2012). Além das redes citadas, podem ser utilizados também os demais vértices que passaram pelo processo de homologação do IBGE. Uma discussão sobre o ajustamento de redes geodésicas objetivando atender os requisitos da Lei 20167 foi apresentada em Silva e Monico (2009). 
Figura 1 - Propriedades georreferenciadas no Brasil. Fonte: INCRA - I3geo.

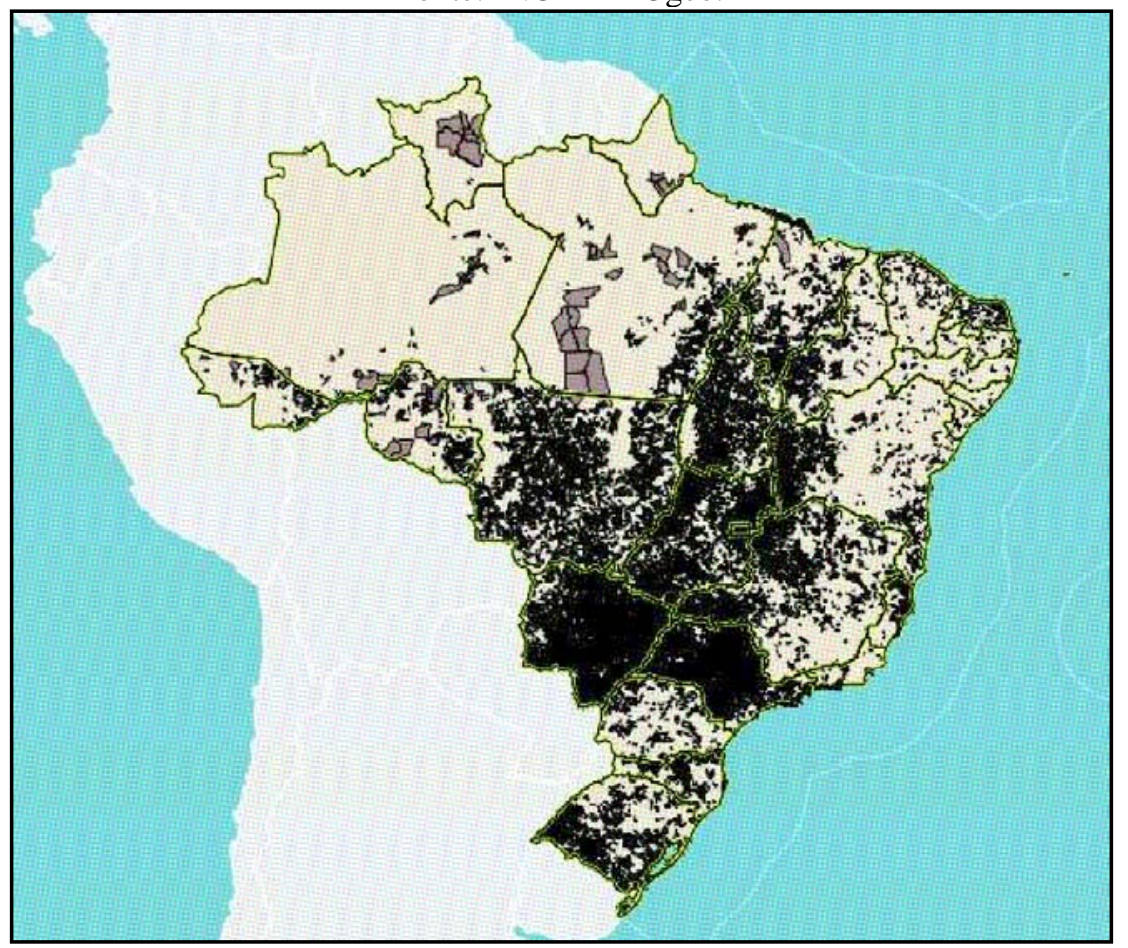

Outro exemplo de empenho no Brasil para a realização do georreferenciamento é um sistema que vem sendo estabelecido pelo INCRA para o processamento on-line dos dados GNSS e a geração de VRS (Virtual Reference Station) na região do levantamento, no entanto, encontra-se indisponível (INCRA 2013). Também, pode ser citado o serviço de PPP (Posicionamento por Ponto Preciso) on-line disponibilizado pelo IBGE (Instituto Brasileiro de Geografia e Estatística), que pode ser utilizado na determinação de pontos de apoio imediato e de limites para o georreferenciamento (IBGE, 2013).

\section{SISTEMAS GNSS}

Muito embora o GNSS seja composto por diversos sistemas, como os sistemas globais e regionais, nesta seção serão apresentados somente o GPS, GLONASS e Galileo.

A descrição completa dos sistemas podem ser obtidas em Seeber (2003), Monico (2008), Hofmann-Wellenhof et al. (2008). Em tais obras pode-se encontrar 
a descrição dos segmentos espaciais, terrestres e usuários de cada sistema. Objetivase aqui apresentar as inovações pelas quais os sistemas vêm passando.

O GPS passa por um processo de modernização, com o acréscimo de novos sinais, a portadora L5 e os códigos L2C e L5C.

O código L2C, modulado na portadora L2, está disponível desde 13 de novembro de 2005, nos satélites do Bloco IIRM. Atualmente, os PRN 17, 31, 12, 15, 29, 07, 01 e 05 transmitem o código L2C. O sinal L2C é dividido em dois códigos de diferentes comprimentos, um de comprimento moderado e outro de comprimento longo. O código CM (Comprimento Moderado) possui comprimento de 10.230 chips, repete a cada 20 milissegundos e é modulado com dados de navegação. O código CL (Comprimento Longo) possui comprimento de 767.250 chips, repete a cada 1,5 segundos e não possui dados modulados sobre o mesmo (FONTANA et al., 2001).

O sinal L5C é transmitido apenas pelo único satélite do Bloco IIF e é um sinal exclusivamente civil, pois sua frequência pertence a ARNS (Aeronautical Radio Navigation Service). Este sinal será amplamente utilizado por usuários do setor de aviação, que utilizarão receptores de dupla-frequência, o que resultará em uma melhora nos resultados em posicionamento (PULLEN; ENGE, 2004).

Desenvolvido pela União Soviética e atualmente sob controle da Rússia, o sistema GLONASS foi declarado operacional em 1995, no entanto, com a falta de lançamento de novos satélites e a curta vida útil dos que estavam em órbita, o número de satélites decresceu, chegando a 7 no final de 2002. A partir de 2004, iniciou-se o lançamento de novos satélites, criando expectativas bastante positivas. Atualmente, a constelação é composta por 31 satélites, sendo que 24 estão operacionais (GLONASS, 2012).

Os satélites GLONASS que serão lançados futuramente, os da série GLONASS-M, fazem uso da técnica CDMA (Code Division Multiple Access) para transmissão dos sinais, sendo que a constelação atual utiliza a técnica FDMA (Frequency Division Multiple Access).

Em desenvolvimento pela ESA (European Space Agency), juntamente com a Comissão Européia e a indústria do mesmo continente, o Galileo é o sistema de navegação por satélites europeus de controle civil, ao contrário do GPS e GLONASS que são militares. A constelação prevista para o sistema possui 30 satélites, sendo 27 operacionais e 3 de reserva, distribuídos em três planos orbitais MEO, com inclinação de $56^{\circ}$ e altitude de $23.600 \mathrm{~km}$. Com esta configuração de constelação, o Galileo apresentará boa cobertura em altas latitudes.

Atualmente o sistema conta com 4 satélites em órbita. O primeiro satélite foi lançado em 28 de dezembro de 2005 e o segundo foi lançado em 27 de abril de 2008 e os dois últimos foram lançados em 21/10/2011. A missão do GIOVE A é assegurar a utilização das frequências atribuídas pela ITU (International Telecommunications Union) para o Galileo, demonstrar as tecnologias essenciais para navegação, caracterizar o ambiente planejado para as órbitas Galileo e testar receptores na Terra (ESA, 2006). Segundo Akos et al. (2006). Ambos os satélites 
transmitem todos os sinais Galileo, no entanto, o GIOVE A transmite cada sinal por vez nas três portadoras e o GIOVE B faz a transmissão simultaneamente. Além disso, ambos transmitem os serviços Galileo para testes e validação.

Os sinais de navegação do Galileo são transmitidos em quatro frequências: E1, E6, E5a, E5b. As frequências selecionadas para o sistema europeu estão alocadas no espectro para RNSS (Radio Navigation Satellite Service), e os sinais E1, E5a e E5b estão alocados no espectro para ARNS (Signal in Space: Interface Control Document, 2010).

A Tabela 1 apresenta um comparativo das frequências dos três sistemas apresentados.

Tabela 1 - Frequência dos sinais GPS (L), GLONASS (G) e Galileo (E).

Fonte: Monico, 2008.

\begin{tabular}{c|c|c|c}
\hline Portadoras & $\begin{array}{c}\text { Abrangência da banda } \\
\text { (MHz) }\end{array}$ & $\begin{array}{c}\text { Múltiplo de } \\
\mathbf{1 0 , 2 3}\end{array}$ & $\begin{array}{c}\text { Frequência } \\
\text { central }\end{array}$ \\
\hline E5a, L5 & $1164-1188$ & 115 & 1176,45 \\
\hline E5b & $1188-1215$ & 117,5 & 1207,14 \\
\hline L2 & $1210-1240$ & 120 & 1227,60 \\
\hline G2 & $1242,937-1247,75$ & & por satélite \\
\hline E6 & $1260-1300$ & 125 & 1278,750 \\
\hline L1, E1 & $1559-1610$ & 154 & 1575,42 \\
\hline G1 & $1598,625-1604,25$ & & por satélite \\
\hline
\end{tabular}

Pode-se observar, na Tabela 1, que as portadoras L1 e L5 do GPS encontramse na mesma frequência que as portadoras E1 e E5a do Galileo, o que pode vir a ser uma fonte de interferência. No entanto, tal fato é eliminado a partir da utilização de técnicas de modulação (MONICO, 2008).

Trabalhos desenvolvidos por Bruyninx (2006) e Lago (2001) apresentam resultados da combinação de dados GPS/GLONASS utilizando receptores em estações de referência.

\section{METODOLOGIA}

\section{1 Área teste}

Os levantamentos foram realizados no Assentamento São Jorge, à margem da Rodovia Olímpio Ferreira Silva (SP 272), km 20, no município de Presidente Bernardes/SP. Trata-se de uma área implantada pela FCT/UNESP, em parceria com o ITESP. A área teste foi dividida em três áreas, sendo que cada uma simula uma propriedade rural. A divisão ocorreu visando simular situações que ocorrem quando se faz levantamentos de imóveis vizinhos, ou seja, quando se faz a junção de diferentes levantamentos, na qual os polígonos têm que apresentar acurácia melhor que $0,50 \mathrm{~m}$. A figura 2 apresenta o imóvel em questão. 
Figura 2 - Área teste e divisões.

Fonte: Adaptado Google Earth.

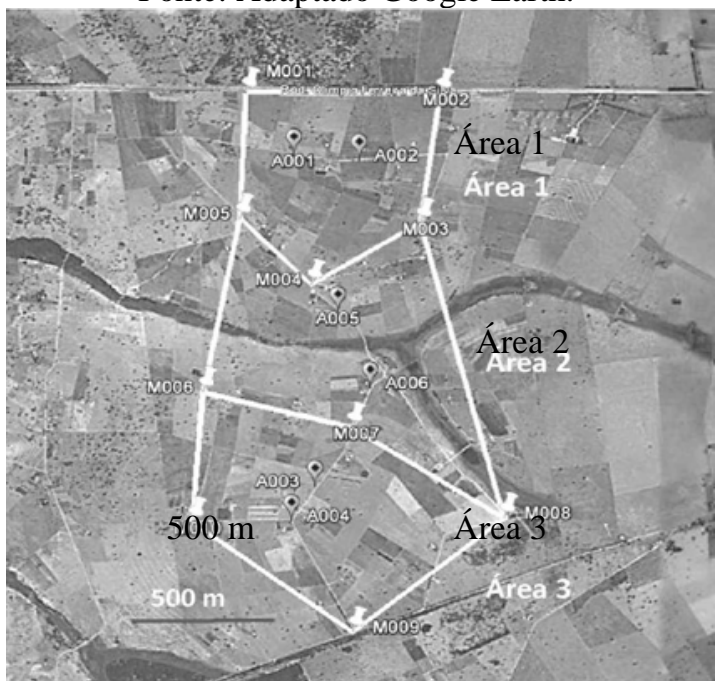

\subsection{Estratégias de coleta e processamento dos dados}

Para o levantamento de campo foram utilizados receptores GNSS Topcon Hiper GGD e Hiper Lite que rastreiam os sinais dos satélites GPS e GLONASS. Tais equipamentos foram configurados com taxa de coleta de 15" e mascara de elevação de $10^{\circ}$. Cada ponto limítrofe foi ocupado por um período igual ou superior a 40 minutos. Já os pontos de apoio básico, foram ocupados por 4 horas.

Segundo INCRA (2011) a finalidade de se determinar um as coordenadas de um ponto de apoio básico é o transporte de coordenadas de vértice de controle planimétrico a partir de dados fundamentais do SGB. Os vértices de apoio básico deverão ser sua posição determinada pelo método relativo estático e a partir de no mínimo dois vértices pertencentes ao SGB.

Os pontos limítrofes, de acordo com INCRA (2011), são os vértices do perímetro do imóvel rural que devem ter suas coordenadas determinadas atendendo a precisão posicional de $0,50 \mathrm{~m}$.

Com o objetivo de avaliar o desempenho da combinação dos dados GPS e GLONASS e cada sistema separadamente, foram realizados diferentes processamentos dos dados coletados em campo.

Para o transporte de coordenadas e determinação dos pontos limítrofes dos imóveis foram definidas as seguintes estratégias:

a) processamentos GPS/GLONASS com efemérides precisas;

b) processamentos GPS/GLONASS com efemérides transmitidas;

c) somente com dados GPS e efemérides precisas; e

d) somente GLONASS e efemérides precisas. 
Dentre todas as estratégias apresentadas, a que utiliza dados GPS/GLONASS e efemérides precisas (estratégia a), foi adotada como referência para avaliação dos demais resultados.

Para o processamento dos dados e ajustamento foi utilizado o software LGO (Leica Geo Office). Como observável, utilizou-se a combinação iono-free, e para correção dos efeitos de troposfera fez-se uso do modelo de Hopfield (LEICA, 2013).

As linhas de base formadas entre as estações da RBMC e pontos de apoio básico possuem comprimento de 34 a $470 \mathrm{~km}$. Para os pontos limítrofes, a média de comprimento das linhas de base é de $1 \mathrm{~km}$.

\section{RESULTADOS}

\subsection{Determinação das coordenadas dos pontos de apoio}

Os dados coletados referentes à ocupação dos pontos de apoio A001 a A006 foram processados e ajustados utilizando as diferentes estratégias de processamento apresentadas anteriormente. Para tal operação foram utilizadas as estações PPTE, ROSA, MSCG e SPCA pertencentes à RBMC, equipadas com receptores capazes de rastrear os satélites das constelações GPS e GLONASS.

Primeiramente, foram processados os dados GPS/GLONASS utilizando efemérides precisas, conforme orienta a NTGIR (Norma Técnica de Georreferenciamento de Imóveis Rurais). Os dados resultantes deste processamento (estratégia a) foram considerados como valores de referência para as demais avaliações. A Tabela 2 apresenta os resultados obtidos.

Tabela 2 - Coordenadas de referência dos pontos de apoio.

\begin{tabular}{c|c|c|c|c|c}
\hline Ponto & $\mathbf{N}(\mathbf{m})$ & $\mathbf{E}(\mathbf{m})$ & $\boldsymbol{\sigma}_{\mathbf{N}}(\mathbf{m})$ & $\boldsymbol{\sigma}_{\mathbf{E}}(\mathbf{m})$ & $\boldsymbol{\sigma}_{\mathbf{p}}(\mathbf{m})$ \\
\hline A001 & 7535431,492 & 428311,710 & 0,004 & 0,008 & 0,009 \\
\hline A002 & 7535393,171 & 428779,671 & 0,006 & 0,012 & 0,013 \\
\hline A003 & 7532871,922 & 428475,411 & 0,015 & 0,048 & 0,050 \\
\hline A004 & 7532606,487 & 428307,222 & 0,008 & 0,045 & 0,046 \\
\hline A005 & 7534214,592 & 428632,808 & 0,007 & 0,016 & 0,017 \\
\hline A006 & 7533637,619 & 428867,642 & 0,008 & 0,018 & 0,020 \\
\hline
\end{tabular}

Como se pode observar na Tabela 2, os pontos (A001 a A006) apresentam precisão planimétrica melhor ou igual a $0,05 \mathrm{~m}$, atendendo a NTGIR, que exige que para os pontos de apoio, a precisão planimétrica deve ser melhor ou igual a 0,10 m.

Posteriormente, foram processados os dados GPS/GLONASS com o uso de efemérides transmitidas (estratégia b). A Figura 3 apresenta os gráficos de discrepância planimétrica e comparação das precisões planimétricas entre os dados de referência e os obtidos com dados GPS/GLONASS e efemérides transmitidas.

Figura 3 - Discrepância planimétrica e comparativo de precisão planimétrica - 
GPS/GLONASS com efemérides transmitidas.

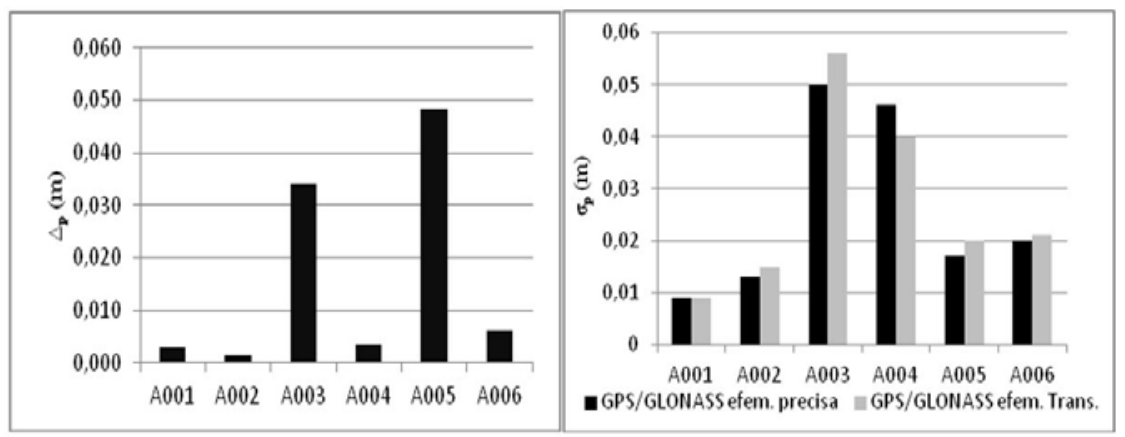

Observa-se na Figura 2 que as discrepâncias são da ordem de centímetros, sendo o menor valor encontrado no ponto A002, que é de 0,001 m e sendo maior no ponto A005, da ordem de $0,048 \mathrm{~m}$. Verifica-se também que os maiores valores são encontrados nos dados obtidos com efemérides transmitidas, com exceção do ponto A004; no entanto, verifica-se a mesma tendência nos valores.

Na terceira estratégia de processamento (estratégia c) dos dados dos pontos de apoio foram utilizados somente dados GPS, juntamente com efemérides precisas. Os gráficos apresentados na Figura 4 trazem as discrepâncias e precisões planimétricas dos pontos.

Figura 4 - Discrepância planimétrica e comparativo de precisão planimétrica - GPS com efemérides precisas.

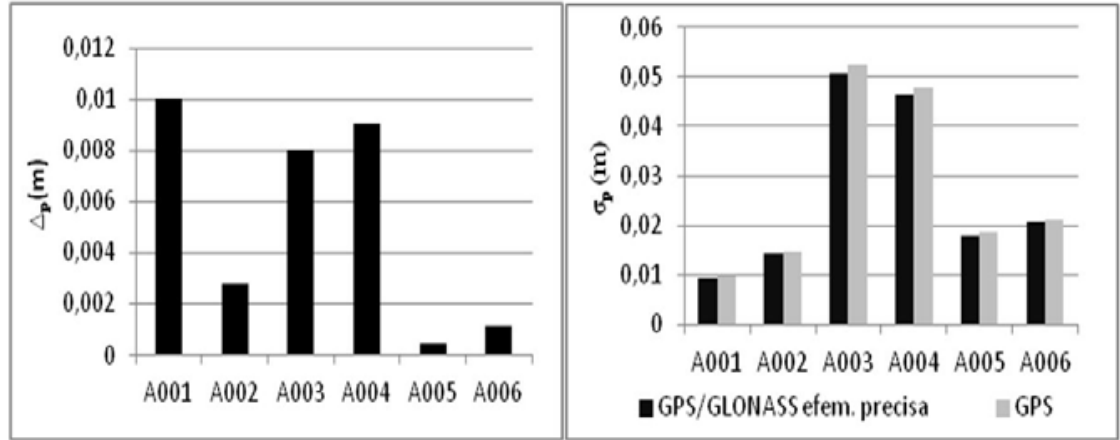

Verifica-se partir da Figura 4 que a discrepância entre as coordenadas de referência e as obtidas com dados GPS são da ordem de milímetros, obtendo os maiores valores nos pontos A001 e A004, $10 \mathrm{~mm}$ e 8,9 mm, respectivamente. Observa-se também que as resultantes de precisão planimétrica das duas estratégias de processamento são insignificantes. Os maiores valores encontram-se no ponto A003, que deve ser devido às árvores de grande porte próximas do ponto. 
Na quarta estratégia (estratégia d) de processamento foram utilizados somente dados GLONASS, também utilizando efemérides precisas. Por meio do gráfico apresentado na Figura 5 pode-se observar as discrepâncias das coordenadas quando comparadas com os dados de referência.

Figura 5 - Discrepância planimétrica - GLONASS com efemérides precisas.

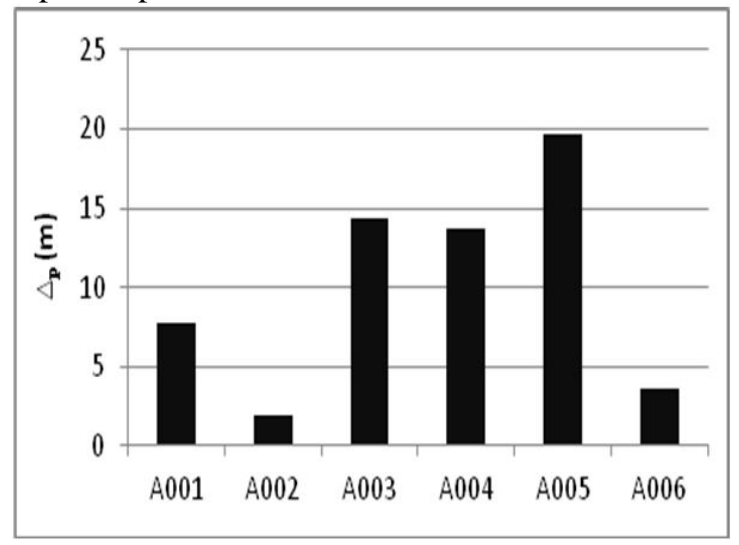

Verifica-se no gráfico apresentado na Figura 5 que as discrepâncias são da ordem de metros, sendo a menor no ponto A002 e a maior no ponto A005, com valores de 1,845 m e 19,630 m, respectivamente.

A diferença de precisão planimétrica tem o maior valor é encontrado no ponto A005, e o menor no ponto A004, sendo 16,170 m e 4,371 m, respectivamente.

\subsection{Determinação dos pontos limítrofes}

Determinadas as coordenadas dos pontos de apoio, realizou-se o processamento dos dados dos pontos limítrofes das áreas. Tais pontos também foram ajustados, visto que cada área possui duas estações de referência.

Na primeira etapa foram processados dos dados GPS/GLONASS utilizando efemérides precisas (estratégia a), os resultados obtidos foram considerados como referência para a avaliação dos demais. A Tabela 3 apresenta os resultados do processamento dos dados de referência.

Verifica-se na Tabela 3 que os desvios-padrão são da ordem de milímetros, sendo o maior valor da componente $\mathrm{N}$ obtido no ponto M002, para a componente E, o maiores valores são obtidos no ponto M004, ao tratar-se da Área 1. Em relação à Área 2, os maiores valores para os desvios-padrão são encontrados no ponto M008. E, para Área 3, o maior valor é encontrado no ponto M008. Deve-se considerar que neste caso, todas as linhas de base apresentaram solução fixa, sendo os valores inteiros das ambiguidades foram estimados. 
Tabela 3 - Coordenadas GPS/GLONASS dos pontos limítrofes - Efemérides precisas.

\begin{tabular}{c|c|c|c|c|c|c}
\hline Área & Ponto & $\mathbf{N}(\mathbf{m})$ & $\mathbf{E}(\mathbf{m})$ & $\boldsymbol{\sigma}_{\mathbf{N}}(\mathbf{m})$ & $\boldsymbol{\sigma}_{\mathbf{E}}(\mathbf{m})$ & $\boldsymbol{\sigma}_{\mathbf{p}}(\mathbf{m})$ \\
\hline Área 1 & M001 & 7535916,668 & 427966,922 & 0,002 & 0,003 & 0,004 \\
\cline { 2 - 7 } & M002 & 7535911,787 & 429358,337 & 0,002 & 0,004 & 0,004 \\
\cline { 2 - 7 } & M003 & 7534913,060 & 429221,805 & 0,002 & 0,003 & 0,004 \\
\cline { 2 - 7 } & M004 & 7534433,959 & 428440,932 & 0,002 & 0,004 & 0,004 \\
\cline { 2 - 7 } & M005 & 7534942,839 & 427928,509 & 0,002 & 0,004 & 0,004 \\
\hline Área 2 & M003 & 7534913,061 & 429221,810 & 0,001 & 0,001 & 0,001 \\
\cline { 2 - 7 } & M004 & 7534433,964 & 428440,944 & 0,002 & 0,001 & 0,002 \\
\cline { 2 - 7 } & M005 & 7534942,820 & 427928,526 & 0,001 & 0,001 & 0,001 \\
\cline { 2 - 7 } & M006 & 7533601,086 & 427666,793 & 0,001 & 0,001 & 0,001 \\
\cline { 2 - 7 } & M007 & 7533330,861 & 428734,007 & 0,001 & 0,001 & 0,001 \\
\cline { 2 - 7 } & M008 & 7532635,366 & 429830,041 & 0,002 & 0,003 & 0,004 \\
\hline Área 3 & M006 & 7533601,072 & 427666,650 & 0,005 & 0,024 & 0,025 \\
\cline { 2 - 7 } & M007 & 7533330,850 & 428733,882 & 0,005 & 0,002 & 0,005 \\
\cline { 2 - 7 } & M008 & 7532635,443 & 429829,143 & 0,009 & 0,053 & 0,054 \\
\cline { 2 - 7 } & M009 & 7531750,016 & 428748,970 & 0,005 & 0,024 & 0,025 \\
\cline { 2 - 7 } & M010 & 7532592,782 & 427600,395 & 0,005 & 0,024 & 0,025 \\
\hline
\end{tabular}

No segundo processamento (estratégia b), semelhantemente ao transporte de coordenadas, efetuou-se o processamento de dados GPS e GLONASS utilizando efemérides transmitidas. Os gráficos apresentados pela Figura 6 apresentam as discrepâncias (direita) dos dados processados com efemérides transmitidas em relação aos processados com órbitas precisas e as precisões de coordenadas de cada processamento.

Figura 6 - Discrepância e precisão planimétrica dos pontos limítrofes.

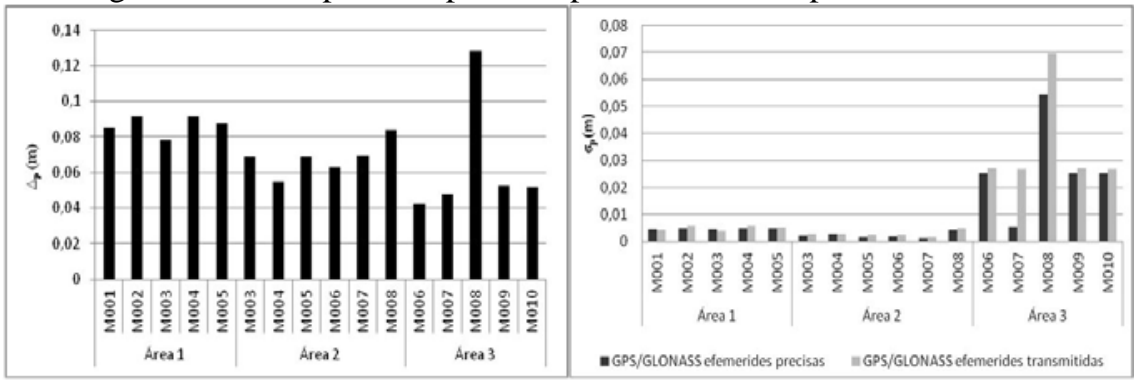

Verifica-se na Figura 6 que as resultantes das discrepâncias planimétricas das coordenadas estão entre $0,042 \mathrm{~m}$, no ponto M006, e 0,128 m para o ponto M008, 
ambos pertencentes à Área 3. A discrepância do ponto M008 não segue a tendência dos demais, que estão entre 0,040 e $0,060 \mathrm{~m}$.

Na Área 1, os pontos determinados a partir de efemérides transmitidas possuem valores maiores para a resultante da precisão planimétrica. Já para a Área 2, nota-se que os resultados de referência são mais precisos que os determinados com efemérides transmitidas. Na área 3 ocorre um aumento nos valores, tanto para os dados de referência como para os processados com efemérides transmitidas. Observa-se também neste caso que as precisões planimétricas de referência são menores que os determinados com efemérides transmitidas, como ocorrido na Área 2.

Posteriormente, foram processados somente dados GPS (estratégia c). Os gráficos da Figura 7 apresentam as discrepâncias e precisões planimétricas em relação aos dados de referência (estratégia a).

Figura 7 - Discrepância planimétrica e comparativo de precisão - GPS com efemérides transmitidas.

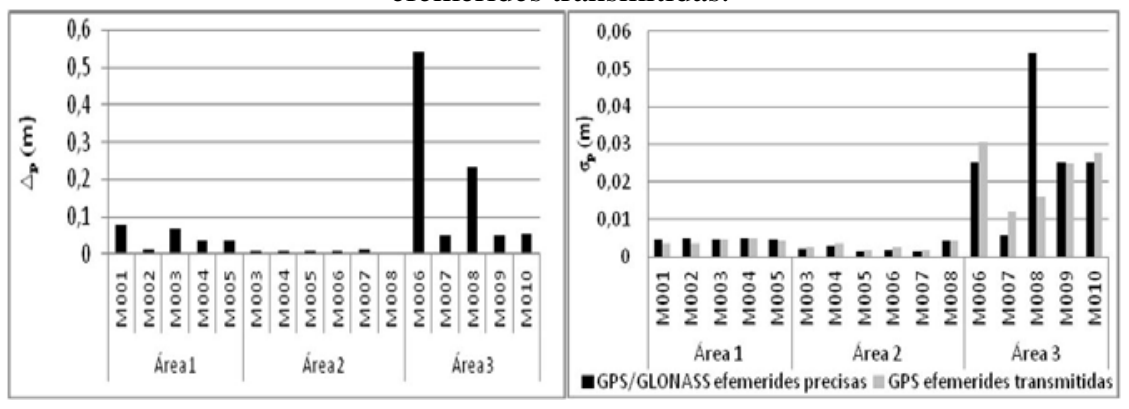

Observa-se na Figura 7 que, a maioria dos pontos apresentam discrepância planimétrica menor que $0,10 \mathrm{~m}$, com exceção dos pontos M006 e M008, ambos referentes à Área 3. Neste caso o ponto M006 apresentou discrepância de 0,540 m e o ponto M008 de $0,230 \mathrm{~m}$. Tais pontos também foram rastreados no levantamento da Área 2, com o mesmo tempo de rastreio, no entanto, em horários diferentes, o que pode indicar alguma alteração relacionada a ionosfera, já que a área 3 foi levantada no final da tarde e início da noite. Tanto para a Área 2 como para a Área 3 foram rastreados entre 7 e 10 satélites para os pontos M006 e M008.

As menores discrepâncias estão na Área 2, que possui pontos comuns com a área 1, que também apresentaram menores discrepâncias.

Verifica-se na Figura 7, para a Área 1, os valores da resultante de precisão planimétrica obtidos com dados GPS são menores que os dados de referência. No entanto, para a Área 2, o resultado inverte, ou seja, as resultantes dos dados de referência são menores que as determinadas a partir de dados GPS, sendo os maiores valores encontrados no ponto M008, igual a 0,004 m, tanto para os dados de referência como os obtidos com GPS. 
Na Área 3, repete-se o cenário ocorrido ao analisar os dados GPS com efemérides transmitidas. Verifica-se um aumento nos valores de precisão planimétrica, sendo que para os pontos M006, M007 e M010 os valores são maiores para os dados GPS, que são da ordem de 0,030 m, 0,012 m e 0,027 m, respectivamente.

No último processamento (estratégia d) dos dados referentes aos pontos limítrofes foram utilizados apenas dados GLONASS. As discrepâncias e precisões em relação aos dados de referência podem ser observadas nos gráficos apresentados pela Figura 8.

Figura 8 - Discrepância planimétrica e comparativo de precisão - GLONASS com efemérides transmitidas.

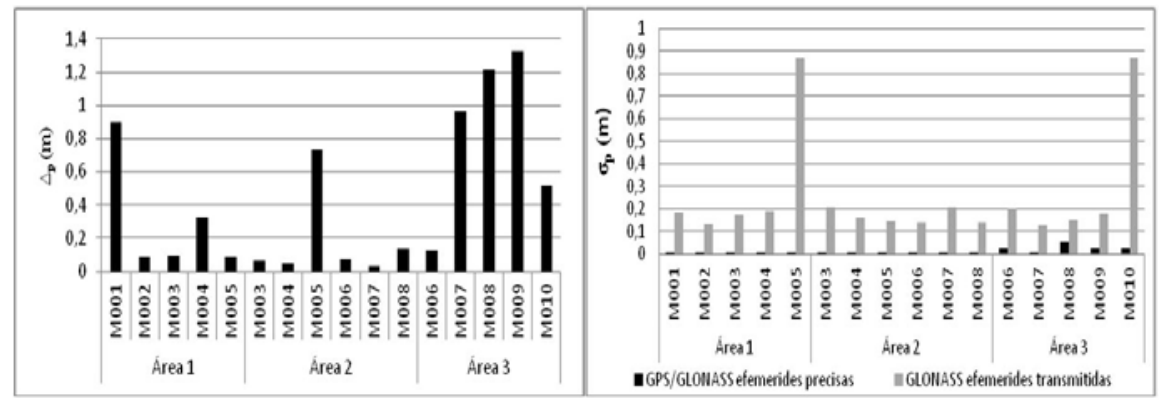

Verifica-se na Figura 8, que para a Área 1, a maior discrepância é obtida no ponto M001, que é igual a 0,890 m. Nota-se também outro pico no ponto M004, de 0,320 m. Para os demais pontos desta área, as discrepâncias são inferiores a 0,200 $\mathrm{m}$, sendo o menor valor pertencente ao ponto M005, que é de 0,080 m.

Para a Área 2, com exceção do ponto M005, cuja discrepância foi de 0,76 m, os demais apresentam discrepâncias menores que um decímetro.

Para a Área 3, verifica-se discrepâncias maiores que um metro para os pontos M008 e M009, 1,220 m e 1,320 m, respectivamente. Sendo a menor discrepância encontrada no ponto M006, que é de $0,120 \mathrm{~m}$.

Avaliando as precisões apresentadas na Figura 8, observa-se a grande discrepância entre os valores, já que, devido à escala, os valores dos dados de referências não são representados.

Para a Área 1 verifica-se que os pontos determinados com GLONASS, com exceção do M005, apresentam precisão planimétrica que atende a exigência do INCRA, ou seja, menor que $0,50 \mathrm{~m}$. No caso da Área 2, um comportamento mais homogêneo entre os pontos, sendo que, todos atenderiam a precisão exigida. Na Área 3 observa-se um comportamento semelhante ao da Área 1, tendo somente um ponto que não atende a exigência da NTGIR, no caso o ponto M010. 
Para a Área 1, o número de satélites GLONASS variou entre 4 e 5 satélites, o que ocorreu também para a Área 2. Observou-se um aumento quantidade de satélites na Área 3, que ficou entre 4 e 6.

\section{CONCLUSÃO}

O principal objetivo deste trabalho foi avaliar o desempenho da combinação de dados GPS e GLONASS e de cada sistema separadamente. Tais avaliações foram feitas considerando a influência desses resultados no georreferenciamento de imóveis rurais, que tem utilizado posicionamento GNSS para a determinação dos limites das propriedades.

Os processamentos realizados com os dados coletados em campo permitiram determinar o desempenho de cada sistema, bem como de suas combinações. Foram realizados processamentos para a determinação de coordenadas de pontos de apoio básico e dos pontos limítrofes das áreas em questão.

Os resultados do processamento de dados GPS/GLONASS com efemérides transmitidas (estratégia b) apresentaram resultados precisos, o ponto A003 foi o que apresentou o maior valor para a precisão planimétrica, da ordem de 0,056 m. Já a discrepância com os dados de referência, o maior valor foi de $0,250 \mathrm{~m}$ no ponto A004. Desta forma, com a precisão atingida, este processamento atende os requisitos da NTGIR.

A segunda estratégia de processamento (estratégia c) utilizou dados GPS e efemérides transmitidas, que apresentaram precisão planimétrica da mesma ordem que os dados de referência. As discrepâncias com os dados de referência foram menores que $0,010 \mathrm{~m}$, valor este atingido somente pelo ponto A001, atendendo a NTGIR.

Na sequência, processaram-se somente dados GLONASS, sendo que nenhum ponto apresentou precisão planimétrica que venha a atender a NTGIR, chegando a 16,17 m. As discrepâncias deste processamento com os dados de referência foram da ordem de 1,845 m a 19,630 m. Em relação à precisão este processamento não atingiu a precisão da NTGIR.

Após os experimentos com a determinação dos pontos de apoio básico, processaram-se os dados referentes aos pontos de limites das áreas, tendo novamente como referência os dados GPS/GLONASS com efemérides precisas. Neste caso, obteve-se precisão melhor que $0,05 \mathrm{~m}$ para os pontos pertencentes às três áreas, atendendo a NTGIR.

Posteriormente, foram utilizados dados GPS/GLONASS com efemérides transmitidas, que apresentaram valores de precisão planimétria próximos aos valores de referência. A discrepância com as coordenadas de referência estão entre 0,040 m e $0,130 \mathrm{~m}$. Neste caso, todos os pontos atenderam ao requisito de precisão da NTGIR.

Na sequência, somente dados GPS e de efemérides precisas foram utilizados. $\mathrm{O}$ resultado que se obteve foram valores menores para as precisões planimétricas em 
relação aos valores de referência, e discrepâncias entre 0,054 m e 0,232 m. Novamente, os resultados atendem a NTGIR.

Com o processamento de dados GLONASS, obtiveram-se discrepâncias planimétricas maiores que $1,00 \mathrm{~m}$. Em relação às precisões, na maioria dos pontos foram menores que $0,200 \mathrm{~m}$, no entanto, nem todos os pontos atendem a NTGIR.

A partir deste estudo conclui-se que o uso de dados GPS e GLONASS atendem as necessidades do georreferenciamento de imóveis rurais no Brasil, seja para o transporte de coordenadas, seja para a determinação dos pontos limítrofes.

Verifica-se também que o uso de efemérides precisas não apresenta grande influência no caso do transporte de coordenadas.

Atestou-se mais uma vez a potencialidade do uso de dados GPS para o levantamento de imóveis rurais. Também se observou que o uso de dados GLONASS, isoladamente, ainda não atendem as exigências para o transporte e na determinação de pontos limítrofes.

\section{REFERÊNCIAS BIBLIOGRÁFICAS}

AKOS, D.; CHEN, A.; DANTEPAL, J.; ENGE, P. GAO, G. X.. GRELIER, T.; ISSLER, J.; LO, S.; RIES, L. GNSS Album: images and spectral signatures of the new GNSS signals. Inside GNSS, Oregon, v. 1, n. 4, p 46-56, mai/jun of 2006

BRASIL. Decreto $\mathrm{n}^{\circ}$ 7.620, de 21 de novembro de 2011. Altera o art. 10 do Decreto no 4.449, de 30 de outubro de 2002, que regulamenta a Lei no 10.267, de 28 de agosto de 2001. Disponível em <http://www.planalto.gov.br>. Acesso em: 15 de janeiro de 2012.

BRASIL. Lei $\mathrm{n}^{\circ} 10.267$, de 28 de agosto de 2001. Altera dispositivos das Leis $\mathrm{n}^{0}$ 4.947, de 6 de abril de 1966, 5.868, de 12 de dezembro de 1972, 6.015, de 31 de dezembro de 1973, 6.739, de 5 de dezembro de 1979, 9.393, de 19 de dezembro de 1996, e dá outras providências. Disponível em <http:// www.planalto.gov.br>. Acesso em: 15 de janeiro de 2010.

BRASIL. Decreto $\mathrm{n}^{\circ} 4.449$, de 30 de outubro de 2002. Dispõe sobre a regulamentação da Lei $\mathrm{n}^{0}$ 10.267/01. Disponível em <http://www.planalto. gov.br>. Acesso em: 15 de janeiro de 2010.

BRASIL. Decreto $n^{\circ} 4.449$, de 31 de outubro de 2005. Dá nova redação aos dispositivos do Decreto ${ }^{\circ} 4.449$, de 30 de outubro de 2002, e dá outras providências. Disponível em <http://www.planalto.gov.br>. Acesso em: 15 de janeiro de 2010.

BRUYNINX, C. Comparing GPS-only with GPS+GLONASS positioning in a regional permanent GNSS network. GPS Solutions, n. 11, p. 97-106. 2007.

ESA. European GNSS Supervisory Authority. Contract for in-orbit validation of Galileo system. Disponível em <http://www.esa.int/SPECIALS/Galileo_ Launch/ SEMAOAMZCIE_0.html>. Acesso em: 01 maio de 2011. 
FONTANA, R. D.; CHEUNG, W.; NOVAK, P. M.; STANSELL, T. A. The new L2 civil signal. 2001. Disponível em: <http://www.navcen.uscg.gov/gps/ modernization/TheNewL2CivilSignal.pdf> . Acesso em 10 de maio de 2011.

GLONASS. Federal Space Agency. Information Analytica Centre. 2012. http://www.glonass-ianc.rsa.ru/pls/htmldb/f?p=201:1:18305357346316216989 Acesso em 5 de maio de 2012.

HOFMANN-WELLENHOF, B.; LICHTENEGGER H.; WASLE. GNSS: Theory and practice. N. York: Springer-Verlag Wien, 2008

IBGE. Sistema Geodésico Brasileiro - Redes Estaduais GPS. Disponível em: http://www.ibge.gov.br/home/geociencias/geodesia/estadual.shtm. Acesso em dezembro de 2012.

IBGE. Posicionamento por Ponto Preciso. Disponível em http://www.ibge.gov.br/ home/geociencias/geodesia/ppp/default.shtm. Acesso em janeiro de 2013.

INCRA. Norma Técnica para o Georreferenciamento de Imóveis Rurais. $1^{\mathrm{a}}$ ed. 2003. Brasília.

INCRA. Norma Técnica para o Georreferenciamento de Imóveis Rurais. $2^{\mathrm{a}}$ ed. revisada 2010. Brasilia Disponível em <http://200.252.80.40/Credencia/ download/Norma_Tecnica.pdf $>$. Acesso em 20 de janeiro de 2012.

INCRA. Rede INCRA de Bases Comunitárias do GNSS - RIBaC. Disponível em: http://ribac.incra.gov.br/. Acessado em 11 de fevereiro de 2013.

LAGO, I. F.; FERREIRA, L. D. D.; KRUEGER, C. P. Integração GPS e GLONASS aplicada aos levantamentos geodésicos. Boletim de Ciências Geodésicas, Curitiba, v. 7, nº1, 2001

LEICA. Leica Geo Office. Disponível em: http://www.leicageosystems.com/ en/Leica-Geo-Office_4611.htm, 2013.

MONICO, J. F. G. Posicionamento pelo GNSS: descrição, fundamentos $e$ aplicações. São Paulo: Editora UNESP, 2008.

PULLEN, S. ENGE, P. A civil user perspective on near-term and long-term GPS modernization. Department of Aeronautics and Astronautics, Stanford University. Disponível: http://waas.stanford.edu/ wwu/papers/gps/PDF/ PullenJapanGNSS04.pdf. Acesso em 10 de maio de2011.

SEEBER, G. Satellite Geodesy: Foundations, Methods and Applications. New York: Walter de Gruyter, 2003.

SILVA, H. A.; MONICO, J. F. G. Ajustamento de Redes GPS em conformidade com as exigências da Lei 10.267/2001. Boletim de Ciências Geodésicas, Curitiba, v. 15, n³, 2009.

(Recebido em agosto de 2012. Aceito em março de 2013.) 